\title{
Évaluation économique et réflexions sur la cohérence des relations helvético-indonésiennes : point de vue d'une banque
}

Max Schieler

\section{CpenEdition} Journals

Édition électronique

URL : http://journals.openedition.org/aspd/1456

DOI : $10.4000 /$ aspd. 1456

ISSN : 1663-9669

Éditeur

Institut de hautes études internationales et du développement

Édition imprimée

Date de publication : 1 février 1995

Pagination : 186-190

ISSN : 1660-5934

\section{Référence électronique}

Max Schieler, «Évaluation économique et réflexions sur la cohérence des relations helvéticoindonésiennes : point de vue d'une banque ", Annuaire suisse de politique de développement [En ligne], 14 | 1995, mis en ligne le 03 mai 2013, consulté le 08 septembre 2020. URL : http:// journals.openedition.org/aspd/1456; DOI : https://doi.org/10.4000/aspd.1456 


\title{
Évaluation économique et réflexions sur la cohérence des relations helvético-indonésiennes: point de vue d'une banque*
}

\author{
Max Schieler, Société de Banque Suisse
}

\section{Evaluation de la solvabilité du pays}

Les explications ci-après concernant l'Indonésie représentent l'esquisse d'une analyse du pays dans l'optique d'une banque. Celle-ci mettra au premier plan l'appréciation de la solvabilité du pays, autrement dit de la capacité et de la volonté du pays de générer suffisamment de devises pour honorer comme il se doit ses engagements financiers à l'étranger. II s'agit là d'un risque qui s'ajoute au risque de solvabilité individuelle dans les affaires réalisées au-delà des frontières et qui ne relève pas du domaine de responsabilité du débiteur réel (à moins que ce débiteur ne soit l'Etat lui-même ou une entreprise d'Etat). II se peut ainsi qu'un crédit accordé à un débiteur domicilié à l'étranger puisse rester impayé, du fait que le pays n'est ni en mesure d'assurer le transfert, ni disposé à l'effectuer. Et ce, même si le débiteur réel est tout à fait solvable (en monnaie locale). L'évaluation de cet élément de risque - importante, par nature, pour toute banque active sur le plan international - est faite à la SBS sur la base d'une série de variables macro-économiques sélectionnées et d'une analyse du risque politique - et ce, toujours par rapport à la capacité et la volonté de payer. L'appréciation du risque qui en résulte participe de la décision lorsqu'il s'agit de définir la politique commerciale adaptée à un pays tel que l'Indonésie, ainsi que l'ampleur, le type et le degré de maturité de son engagement. A cet égard, l'analyse ne doit pas, de manière unilatérale, mettre en évidence uniquement les risques, mais aussi les chances éventuelles (le potentiel).

Une évaluation de l'Indonésie effectuée sous cet angle montre que, vu son développement économique fulgurant au cours de ces 25 dernières années, ce pays peut être considéré à juste titre comme un enfant du "East Asian Economic Miracle». Même si l'appréciation du "miracle économique» indonésien peut différer selon les points de vue, on ne peut que relever objectivement des succès incontestables: par exemple un recul du nombre d'habitants vivant dans la pauvreté (selon la définition de l'ONU) de 70 millions en 1970 à 27

* Les opinions exprimées dans cet article représentent le point de vue de leur auteur et peuvent très bien différer de celles de la Société de Banque Suisse. 
millions aujourd'hui; une hausse de l'espérance de vie moyenne de 11 ans - qui passe de 50 à 61 ans -, une réduction du taux d'analphabétisme de 39 à $16 \%$ et, dès 1984, l'autosuffisance en ce qui concerne la production de riz, aliment de base principal. Dans le même laps de temps, l'Indonésie est entrée dans le club des pays à revenu moyen (autrement dit des pays ayant un PIB par habitant supérieur à $675 \$$ ), ce qui s'est traduit par une augmentation du revenu annuel par habitant qui est passé de $70 \$$ en 1970 à $700 \$$ en 1993. Selon des estimations de la Banque mondiale, dans une comparaison sur la base de la parité du pouvoir d'achat, le revenu annuel par habitant serait en fait quatre fois plus élevé - à savoir: 2970 dollars pour 1992 -, un chiffre effectivement plus proche de la réalité. Enfin, l'Indonésie a réussi à sortir du cercle vicieux de l'endettement et à éviter un rééchelonnement de la dette - tout au moins jusqu'ici -, cet aspect étant bien entendu mis en valeur du côté des banques.

Pour la perspective à moyen et à long terme de ce pays, on peut relever comme point positif le fait que l'Indonésie soit parvenue, grâce aux réformes structurelles de ces dernières années, à créer une base de croissance solide pour son économie. Elle a notamment réussi à diversifier sa production et à réduire sensiblement la dépendance par rapport au secteur du pétrole et du gaz. $\mathrm{Si}$, au début des années 80 , ce secteur représentait encore $70 \%$ des recettes de l'Etat et $80 \%$ des gains réalisés à l'exportation, il ne lui en incombe aujourd'hui plus que le quart pour chacun de ces postes budgétaires. C'est surtout à la suite de la chute vertigineuse des prix du pétrole en 1986 que l'Indonésie a fortement intensifié ses efforts de diversification et activé en toute logique ses exportations des produits autres que le pétrole et le gaz. Au cours de ces dernières années, les nouvelles branches d'exportation sont d'ailleurs devenues le moteur de la croissance élevée. A ces facteurs s'ajoute la stricte discipline fiscale de l'Indonésie, même si, par le passé, les budgets officiellement déclarés et toujours équilibrés résultaient en premier lieu d'une définition particulière. Selon le système de budgétisation indonésien, les moyens provenant de l'aide au développement sont précisément comptabilisés comme recettes de l'Etat, ce qui permet généralement de compenser les montants déficitaires. C'est à cela que l'on peut mesurer le puissant soutien apporté à l'Indonésie par des donateurs étrangers, notamment par le "Consultative Group for Indonesia" (CGl), placé sous la houlette de la Banque mondiale. Les institutions internationales et les pays donateurs représentés au sein de cette organisation d'entraide ont accordé une nouvelle fois à l'Indonésie et pas plus tard qu'en juillet 1994 des moyens d'un montant de 5,2 milliards de dollars pour l'année fiscale 1994/95 (après $\$ 5,1$ milliards pour 1993/94), rendant une fois de plus hommage à la politique économique rigoureuse menée par Djakarta. Le recul des taux de croissance observé en 1993 à l'exportation et dans les investissements à l'étranger a incité le gouvernement à décider en juin 1994 de deux nouveaux trains de mesures de déréglementation concernant la libéralisation du commerce, en particulier les investissements directs. Un exemple qui illustre une fois de plus la manière pragmatique dont le gouvernement réagit à de nouveaux défis ou à des difficultés économiques. 
Nonobstant les conflits acharnés déclenchés en vue d'organiser la promotion industrielle, il ne faut pas s'attendre en premier lieu à ce que l'on s'écarte des objectifs fondamentaux de la politique économique - assurer la stabilité macroéconomique, l'ouverture vers l'extérieur et la lutte contre la pauvreté. Le rôle toujours prioritaire que joue la stabilité dans l'ensemble de l'économie se traduit également par la manière dont évolue la politique monétaire et fiscale, plus restrictive par rapport à l'année précédente (1993). Celle-ci s'est traduite par une limitation réaliste de l'inflation et du déficit budgétaire qui ont tous deux atteint un niveau acceptable. Cela aura du reste un effet bénéfique sur la balance des paiements, sans que le pays en souffre trop sur le plan de l'évolution de sa croissance. Dans l'ensemble, les perspectives à court terme semblent relativement favorables et, si l'on considère l'avenir à moyen terme, la solvabilité de l'Indonésie ne paraît pas sérieusement menacée.

Si l'on parvient à venir à bout des problèmes latents, le pragmatisme et le maintien opiniâtre de la stabilité continueront, au demeurant, de revêtir une importance primordiale dans la politique économique. Il en est ainsi de la dette extérieure - devenue une charge considérable - qui atteindra dans un avenir proche le seuil des 100 milliards de dollars US et qui limite dès lors énormément la liberté de manoeuvre en matière de politique économique. Le niveau d'endettement de l'Indonésie expose beaucoup plus le pays aux fluctuations du cours du change et des taux d'intérêt, comme cela est récemment ressorti avec netteté une fois encore lors de la forte réévaluation du yen (près de la moitié de la dette extérieure est en yens). A plus long terme, l'Etat devra bien engager une réforme du secteur public, encore très important, mais inefficace, s'il veut réaliser l'objectif de croissance - d'une moyenne de 6,2\% par an - prévu pour le plan quinquennal 1994/99 amorcé en avril dernier. Cela représente un impératif si l'on doit créer un nombre suffisant d'emplois pour la population active qui s'accroît chaque année de 2,5 mio. de personnes, donc la condition sine qua non du maintien de la stabilité sociale et politique.

Malgré l'impressionnant progrès économique, le social gap - le fossé entre les riches et les pauvres - recèle des risques certains d'explosion politique et sociale. C'est ce qu'ont nettement montré les événements du printemps dernier, lors desquels les mouvements de grève des travailleurs demandent des salaires plus élevés ont dégénéré en agressions violentes contre la minorité chinoise, qui ne compte que 6 millions d'âmes, mais occupe une position économique dominante. Cela n'a pas seulement éveillé un certain doute quant à la prétendue stabilité politique du pays, mais donne une idée claire des conflits ethniques, religieux et sociaux qui enflent sourdement sous une façade rassurante. Le maintien de la stabilité est donc également une préoccupation permanente pour le régime qui vient de répondre par une répression accrue à la multiplication des critiques qui s'expriment de plus en plus publiquement. Et cela s'explique également par l'incertitude grandissante que suscite la succession du Président Suharto qui souhaite quitter définitivement ses fonctions en 1998. C'est ce chapitre qui présente les plus grands risques pour un développement économique sain du pays. La question toujours non résolue de la 
succession de Suharto rend extrêmement difficile un pronostic à peu près sûr concernant l'évolution de ce pays à plus long terme. Nous sommes d'avis que la principale question qui se pose aujourd'hui est de savoir si Djakarta ne va pas (ne doit pas) s'écarter à l'avenir de sa ligne actuelle, fructueuse sur le plan de sa politique économique, ce qui pourrait remettre en cause sa position présente de bon débiteur. Certes, plusieurs indices laissent penser que les héritiers de Suharto poursuivront, le moment venu, la politique engagée par leur prédécesseur, mais ils seront confrontés à un délicat exercice d'équilibre entre l'ouverture démocratique et la répression, lequel pourrait avoir facilement un impact négatif sur l'économie.

\section{Quelques remarques sur le problème de la cohérence}

Même si j'estime ne pas être en mesure d'aborder dans le détail la question de la cohérence, resp. de l'incohérence, dans le cas concret des relations entre la Suisse et l'Indonésie, j'aimerais néanmoins livrer quelques réflexions personnelles sur cette problématique. II est possible que l'évaluation que je viens de faire de l'Indonésie ne suscite pas partout la même approbation et soit, par ailleurs, considérée comme étant trop partisane. Comme je l'ai mentionné d'emblée, il s'agit là du point de vue d'une banque, autrement dit d'un représentant de l'économie privée, pour laquelle le développement économique positif et la croissance rapide du marché potentiel occupent, par nature, le tout premier plan. Dans cette perspective, la situation politique est jugée en priorité par rapport aux conditions de base économiques qui en résultent. Ainsi, il est clair que les mesures accrues - et en partie répressives - prises récemment par le régime pour maintenir la stabilité suscitent pour l'instant l'approbation, étant donné que l'instabilité ou le chaos aurait des effets encore plus néfastes, même si l'actuelle manière d'agir du gouvernement indonésien pourrait parfaitement déboucher, à plus long terme, sur le contraire du but recherché.

II ne faut pas se cacher qu'avec une telle optique de la situation - qui privilégie l'intérêt commercial - on déclenche un conflit de finalités entre les intérêts de l'économie privée d'une part, et l'encouragement à la démocratisation du pays et au respect des droits de l'homme d'autre part. Nous avons là un exemple-type du problème de la cohérence dans les relations de la Suisse avec un pays tel que l'Indonésie. II est certain que les atteintes aux droits de l'homme ne doivent pas non plus être simplement justifiées par l'économie privée comme un mal nécessaire lié à la spécificité du pays, pas plus qu'un gouvernement autoritaire qui s'appuie encore de fait sur une structure féodale ne doive recevoir un soutien particulier (sous la forme, par exemple, de livraisons d'armes). Au demeurant, on peut légitimement se demander de quelle manière il serait possible de remplir le mieux possible ces deux exigences. Car il va de soi que des structures démocratiques ne garantissent pas automatiquement le progrès économique et peuvent même, dans une certaine phase de développement, le retarder. II apparaît de même évident que des structures démocratiques ne peuvent perdurer en soi si le succès économique n'est pas 
au rendez-vous. Pour toutes ces raisons, il me paraît irréaliste et inconvenant, par exemple dans le cas de l'Indonésie, d'appliquer des critères occidentaux et de vouloir lui imposer "du jour au lendemain" la démocratie.

Mais cela ne veut bien sûr pas dire qu'il ne faudrait pas soutenir la mise en place de conditions de base démocratiques. Du côté officiel comme du côté privé, l'on doit et l'on peut dénoncer - à chaque fois que l'occasion s'en présente - des pratiques antidémocratiques et de réelles atteintes aux droits de l'homme, sans pour autant vouloir utiliser comme moyen de pression les relations économiques ou l'aide au développement. Quoi qu'il arrive, il peut être judicieux de lier ces relations et cette aide à certaines conditions, en accord avec les institutions internationales et d'autres pays donateurs. En particulier, j'ai quelque doute quant à l'efficacité de sanctions économiques et/ou d'une réduction, voire d'une annulation de l'aide au développement qui seraient décidées unilatéralement par la Suisse. Notre pays est en effet un partenaire commercial trop insignifiant pour l'Indonésie, comme le montrent très bien les expériences faites dans ce domaine par les Pays-Bas. II est probable que l'Indonésie réagirait à de telles tentatives de pression uniquement dans le cadre d'une action concertée des principales nations industrielles et il resterait encore à prouver que cette réaction aille dans le sens souhaité... II n'est pas exclu que, dans pareil cas, l'Indonésie se réfugie dans l'isolement et un rejet des autres pays, ce qui n'améliorerait ni la situation des droits de l'homme, ni la situation économique de la population indonésienne. Que l'on se souvienne de l'exemple peu glorieux de la Birmanie.

L'exemple offert par les pays voisins du sud-est asiatique, tels que la Corée du Sud ou Taiwan, montre par ailleurs qu'un développement économique positif peut très bien entraîner une ouverture en douceur sur le plan politique. C'est, dans un certain sens, logique: la répression politique et le contrôle autoritaire ne s'accordent guère, en effet, avec une économie toujours plus complexe et une interdépendance internationale croissante. A mon avis, une libéralisation économique doit être également suivie, avec le temps, de réformes politiques, faute de quoi la poursuite du progrès économique se trouve remise en cause ainsi que, au bout du compte, la légitimité d'un régime. C'est pourquoi j'estime qu'à plus long terme, l'économie privée a tout intérêt à ce que le progrès économique soit conforté et, partant, sauvegardé par la démocratisation. Compte tenu de cet intérêt, il est tout à fait possible d'apporter à nos relations un "complément" adapté, par exemple, à travers un renforcement de notre coopération dans le domaine de l'aide suisse au développement ou à travers des prises de position "morales" (concernant les droits de l'homme, la démocratie, etc.) lors des contacts pris aux échelons les plus divers. II faut toutefois être conscient que, vu les divergences entre les intérêts à court terme des uns et des autres, il ne sera pas possible d'éviter complètement à l'avenir des conflits de finalités et des contradictions. 